\title{
Star formation in the Eagle Nebula and NGC 6611
}

\author{
J. M. Oliveira, R. D. Jeffries and J. Th van Loon
}

Astrophysics Group, Lennard-Jones Laboratories, Keele University, UK

\begin{abstract}
We present $I Z J H K L^{\prime}$ photometry of the core of the cluster NGC 6611 in the Eagle Nebula. This photometry is used to constrain the Initial Mass Function (IMF) and the circumstellar disk frequency of the young stellar objects. Optical spectroscopy of 258 objects is used to confirm membership and constrain contamination as well as individual reddening estimates. Our overall aim is to assess the influence of the ionizing radiation from the massive stars on the formation and evolution of young low-mass stars and their disks. The disk frequency determined from the $J H K L^{\prime}$ colour-colour diagram suggests that the ionizing radiation from the massive stars has little effect on disk evolution (Oliveira et al. 2005). The cluster IMF seems indistinguishable from those of quieter environments; however towards lower masses the tell-tale signs of an environmental influence are expected to become more noticeable, a question we are currently addressing with our recently acquired ultra-deep (ACS and NICMOS) HST images.
\end{abstract}

Keywords. stars: pre-main-sequence; stars: luminosity function, mass function; stars: formation; circumstellar matter
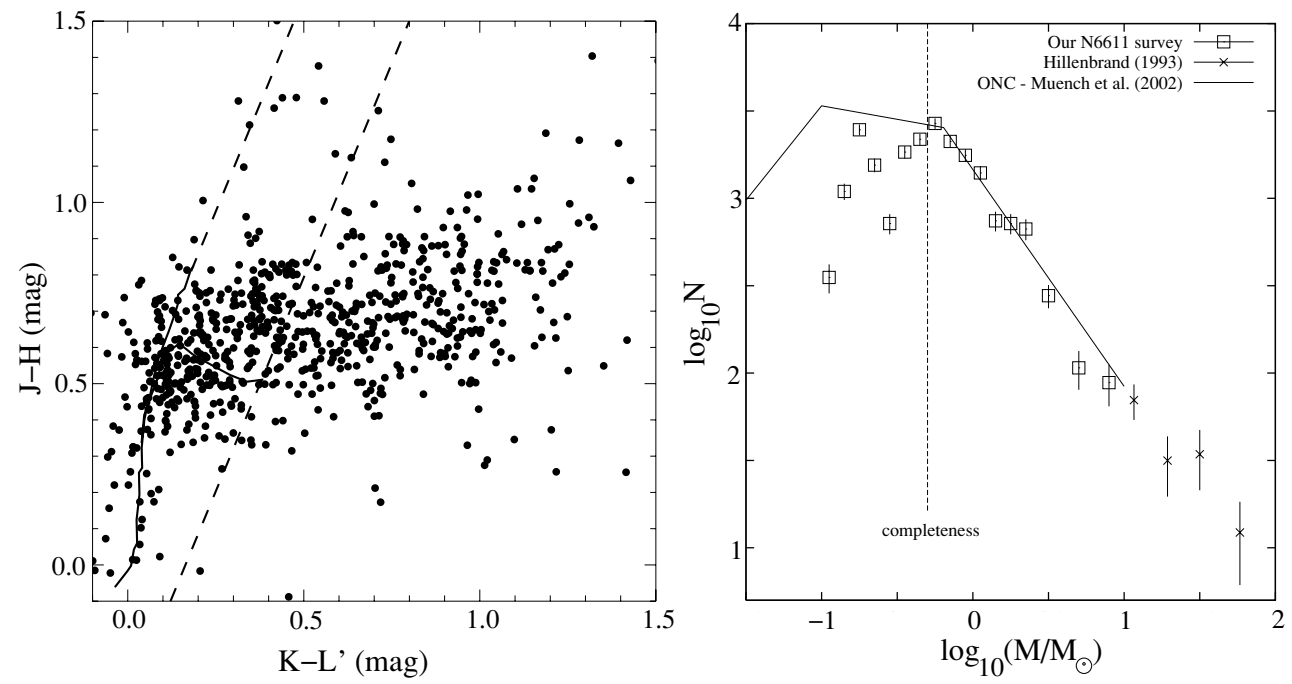

Figure 1. $J H K L^{\prime}$ colour-colour diagram and IMF for NGC 6611. Down to objects of $\sim 0.5 \mathrm{M}_{\odot}$, neither the disk frequency nor the IMF show significant evidence for a strong ionizing influence from the massive O-stars in the cluster (Oliveira et al. 2005).

\section{References}

Hillenbrand, L.A. et al., 1993, ApJ 106, 1906

Muench, A.A. et al., 2002, ApJ 573, 366

Oliveira, J.M. et al., 2005, MNRAS 358, L21 